\title{
EDUCACIÓN SUPERIOR Y SEGREGACIÓN DE GÉNERO: \\ ÓRGANOS UNIPERSONALES DE LA UNIVERSIDAD DE LEÓN
}

\author{
Adelina Rodríguez Pacios \\ Universidad de León
}

\section{Introducción}

En estos días estamos asistiendo a diferentes debates en torno a la Educación Superior. Se trata de homologar las titulaciones universitarias españolas con las del resto de los países de la Unión Europea. Todo parece indicar los cambios deben concluirse para el año 2010, aunque algunas titulaciones ya estarán adaptadas para el año 2007.

Igualmente hemos asistido a cambios legislativos en materia de educación universitaria: en el año 2001 se aprueba la Ley de Ordenación Universitaria (LOU), que más tarde sufre algunas modificaciones, lo que obliga a que cada universidad redacte sus propios estatutos, que suponen asimismo nuevos cambios. Todo parece indicar que nos encontramos en un período de profundos cambios en el ordenamiento universitario español. Pero todo cambia para que no cambie nada. Nos referimos al conservadurismo existente en las estructuras académicas que siguen reflejando la subrepresentación femenina en los órganos de gestión y decisión.

Formalmente, esta es la institución más igualitaria que existe, pero los datos y la realidad son tozudos y demuestran una y otra vez la invisibilidad de las mujeres en la Universidad. 
En este artículo pretendemos describir y analizar la presencia o ausencia de las docentes e investigadoras en los órganos de gobierno en la Universidad de León. En un escrito anterior (publicado en el número 0 de esta revista) hicimos una breve introducción de este tema, pero en ese momento nos encontrábamos con un gobierno universitario en funciones y se preveían cambios, aunque no se produjeron en la dirección deseada. Ahora nos proponemos investigar la existencia de segregación vertical y segregación horizontal entre el personal docente e investigador. Concretamente analizar estos dos tipos de segregación entre los órganos unipersonales de gobierno, de representación y consulta; de las Facultades y Escuelas y de los Departamentos de la Universidad de León.

Efectivamente, como sucede en todas las universidades españolas y de la Unión Europea, las mujeres están subrepresentadas en el grupo de Personal Docente e Investigador de la Universidad de León. Pero su presencia todavía es menor en las categorías docentes superiores. Esta invisibilidad tiene repercusión en los puestos de responsabilidad de la universidad. Este hecho lo avala diferentes estudios realizados en el ámbito universitario y científico (Fernández Villanueva, 1989; García de León, García de Cortázar, 1992; Almarcha Barbado, González Rodríguez, Gonzáles Jorge, 1994; Roca i Tría, 1995; García de Cortázar, García de León, 1997; Instituto de la Mujer, 2001; Antón Sevilla, 2005).

Nos proponemos realizar un análisis de datos secundarios procedentes de la página web de la Universidad de León, recogidos en el año 2005, con el objetivo de determinar la existencia de desigualdades en cuanto al grado de participación en los cargos de responsabilidad, de representación y de gestión en la universidad.

\section{El personal docente e investigador de la Universidad de León}


Educación superior y segregación de género: órganos unipersonales...

Tabla 1

Profesores y Profesoras de la ULE por categoría docente

\begin{tabular}{|l|l|l|l|l|l|l|l|l|}
\hline Categoría & $\mathrm{H}$ & $\mathrm{M}$ & Total & $\% \mathrm{~V}$ & $\% \mathrm{M}$ & Total & $\% \mathrm{~V}$ & $\% \mathrm{M}$ \\
\hline CU & 96 & 18 & 114 & 16,41 & 4,99 & 12,05 & 84,21 & 15,79 \\
TU & 162 & 136 & 298 & 27,69 & 37,67 & 31,50 & 54,36 & 45,64 \\
CEU & 44 & 24 & 68 & 7,52 & 6,65 & 7,19 & 64,22 & 35,78 \\
TEU & 78 & 66 & 144 & 13,33 & 18,28 & 15,22 & 54,18 & 45,82 \\
AU (LRU) & 2 & 4 & 6 & 0,34 & 1,11 & 0,63 & 33,33 & 66,67 \\
AEU (LRU) & 10 & 14 & 24 & 1,71 & 3,88 & 2,54 & 41,68 & 58,32 \\
AYU (LOU) & 17 & 16 & 33 & 2,91 & 4,43 & 3,49 & 51,53 & 48,47 \\
ASOTC & 12 & 18 & 30 & 2,05 & 4,99 & 3,17 & 40 & 60 \\
ASOTP & 157 & 64 & 221 & 26,84 & 17,73 & 23,36 & 71,04 & 28,96 \\
P.EMERITO & 5 & 1 & 5 & 0,85 & 0,28 & 0,53 & 100 & 33,32 \\
P.VISITANTE & 2 & & 3 & 0,34 & & 0,32 & 66,68 & \\
\hline TOTAL & 585 & 361 & 946 & 100 & 100 & 100 & 61,85 & 38,15 \\
\hline
\end{tabular}

Fuente: Universidad de León. Elaboración propia.

$\% \mathrm{~V}$ : Porcentaje de varones en cada categoría con respecto al total de varones.

$\% \mathrm{M}$ : Porcentaje de mujeres en cada categoría con respecto al total de mujeres.

Total: Porcentaje de mujeres y varones en cada categoría. $\% \mathrm{~V}$ : Porcentaje de varones sobre el total de varones y mujeres en cada categoría. $\% \mathrm{M}$ : Porcentaje de mujeres sobre el total de varones y mujeres en cada categoría.

Brevemente queremos recordar cuál es la situación de las profesoras en la ULE por categoría docente. Sabemos que la presencia o ausencia de las mujeres en determinados escalafones de la pirámide universitaria incide de forma directa en la posesión de cargos de responsabilidad. Aunque también somos conscientes de la influencia de otro tipo de variables en la decisión que toman las mujeres de formar parte de los órganos de gestión universitaria.

En el año 2004, el 38,15\% del Personal Docente e Investigador (PDI) de la ULE está formado por mujeres. Es un dato positivo comparado con el conjunto de las universidades españolas, el 33\%, o con el dato de presencia femenina en la universidad de la Unión Europea, el 26\% (CES 3/ 2003: 233). 
Si analizamos la distribución de profesores y profesoras según la categoría docente, apreciamos un desigual reparto, lo que se denomina segregación vertical. Es decir, las mujeres se aglutinan en aquellas categorías docentes que están más alejadas de la cima de la pirámide. Así vemos que componen los porcentajes más elevados en las categorías de Ayudante y Asociados a Tiempo Completo. Están subrepresentadas en los rangos de Catedráticos/as de Universidad, Catedráticos/as de Escuela Universitaria y Asociados/as a Tiempo Parcial (En este último caso por motivos diferentes a los anteriores). Sin embargo, tanto entre los/as Titulares de Universidad como entre los/as Titulares de Escuela Universitaria existe paridad (45,64\% y 45,82\%, respectivamente). En la IV Conferencia Mundial sobre las mujeres, que se celebró en 1995 en Pekín, se acuerda utilizar el concepto de igualdad en la toma de decisiones o la idea de una representación equilibrada de las mujeres, cuando éstas están presentes en no menos del $40 \%$ y no más del 60\%. Esta idea ha servido como referencia para valorar los niveles de participación política y social de las mujeres. Por su parte el Consejo de la Unión Europea, apoyándose en esta idea de representación equilibrada, establece que el umbral mínimo hacia la paridad se consigue cuando se sobrepasa el 30\% de presencia femenina. (CES 3/2003)

La categoría docente superior en la carrera profesional universitaria es la de Catedrático/a de Universidad. Ocupar es estatus en la organización académica es el paso previo para desempeñar algunas tareas de responsabilidad, como por ejemplo la tarea de rector/a. Las profesoras están escasamente representadas en este estrato. Se puede aducir que éste junto con el de Catedráticos/as de Escuela Universitaria son los rangos universitarios menos frecuentados por el profesorado. Pero pensamos que existen algunos mecanismos dentro de la universidad que impiden que un número mayor de mujeres accedan a este estatus. Es el fenómeno denominado "techo de cristal", que hace difícil que las mujeres traspasen la barrera de las categorías docentes de Titular de Universidad o Titular de Escuela Universitaria, en las que sí existe paridad.

\section{Mujeres en los órganos unipersonales de gobierno, de representación y consulta}


Educación superior y segregación de género: órganos unipersonales...

\section{de la Universidad}

a) El Rector. (Capítulo II, Sección 2a, Subsección $1^{\mathrm{a}}$, Estatuto ULE). Según el artículo 79 de los Estatutos "el Rector será elegido por la comunidad universitaria, mediante elección directa y sufragio universal libre y secreto, entre funcionarios del cuerpo de catedráticos de universidad, en activo, que presten sus servicios a tiempo completo en la Universidad de León". La máxima autoridad académica y administrativa de la ULE es un varón. En la actualidad hay tres rectoras en las 71 universidades públicas y privadas españolas. Esta representación de mujeres se asemeja a la de las universidades francesas y alemanas, donde la cifra oscila entre el 4,5\% y el $5 \%$, respectivamente. Sin embargo en las universidades de Suecia, el 18\% de los/as rectores/as son mujeres. (Antón Sevilla, 2005:17)

En una universidad, como la ULE, en la que el $15,79 \%$ del cuerpo de catedráticos de universidad son mujeres, la posibilidad de que una de ellas se presente y gane las elecciones a rector es muy pequeña.

Tabla 2

Órganos unipersonales de gobierno de la ULE

\begin{tabular}{|l|l|l|l|l|l|}
\hline & $\mathrm{V}$ & $\mathrm{M}$ & Total & $\% \mathrm{~V}$ & $\% \mathrm{M}$ \\
\hline Rector & 1 & & 1 & 100 & \\
Vicerrector/a & 9 & 1 & 10 & 90 & 10 \\
Secretariados & 24 & 9 & 33 & 72,73 & 27,27 \\
Secretario/a & 1 & 1 & 1 & 100 & 100 \\
Vicesecretario/a & 1 & 1 & 1 & 100 & 50 \\
Gerente/a & 1 & & 1 & 50 & \\
Vicegerentes/as & & & 2 & & \\
\hline TOTAL & 37 & 12 & 49 & 75,52 & 24,48 \\
\hline
\end{tabular}

Fuente: Universidad de León. Elaboración propia. 
b) Vicerrectores. (Capítulo II, Sección 2a, Subsección 2a , Estatuto ULE). Estos/as son designados y nombrados por el rector entre los/as profesores/as doctores/as que presten sus servicios en la Universidad con dedicación a tiempo completo. Son los responsables de las áreas universitarias que el rector les atribuya (Artículo 81). Los/as vicerrectores/as proponen al rector el nombramiento de los directores de Secretariado, que auxiliarán a éstos/as en el ejercicio de sus funciones. La ULE cuenta con diez vicerrectorados:

* Vicerrectorado de Asuntos Económicos:

- Secretariado de Inversiones.

- Secretariado de Promoción Empresarial.

- Director Gabinete de Imagen Promocional.

* Vicerrectorado del Campus de Ponferrada:

- Secretariado de Coordinación de Investigación.

- Servicio de Radio Universitaria y Actividades Estudiantiles.

- Servicio de Movilidad.

- Servicio de Actividades Físico-Deportivas y Tiempo libre.

* Vicerrectorado de Estudiantes y Asuntos Sociales:

- Secretariado de Asuntos Sociales.

- Secretariado de Deportes y Tiempo Libre.

- Secretariado de Actividades del Alumnado.

- Secretariado de Cooperación educativa y prácticas en la empresa.

- Servicio de Casa del Estudiante.

- Secretariado de Intercambio Nacional de Alumnos.

- Secretariado de Radios Universitarias.

* Vicerrectorado de Innovación Tecnológica:

- Secretariado del CRAI.

- Secretariado de Publicaciones.

- Secretariado de Infraestructuras Tecnológicas.

- Coordinador académico para la difusión de las TIC. 
Educación superior y segregación de género: órganos unipersonales...

* Vicerrectorado de Investigación:

- Secretariado de Institutos Universitarios y Asociaciones de Investigación.

- Secretariado de Gestión de la Investigación.

- Secretariado de Apoyo a la Investigación.

* Vicerrectorado de Ordenación Académica:

- Secretariado de Organización Académica.

- Secretariado de Estudios y Titulaciones.

* Vicerrectorado de Planificación y Evaluación:

- Oficina de Evaluación y Calidad.

*Vicerrectorado de Profesorado:

- Secretariado de Profesorado.

* Vicerrectorado de Relaciones Institucionales y Extensión Universitaria:

- Secretariado de Actividades Culturales y Artísticas.

- Programa Interuniversitario de la Experiencia.

- Secretariado de Cursos y Programas de Extensión Universitaria.

- Centro de Idiomas.

- Secretariado de Relaciones Institucionales.

- Secretariado de Proyección Universitaria.

* Vicerrectorado de Relaciones Internacionales:

- Oficina de Relaciones Internacionales.

- Secretariado de Iberoamérica.

Sólo uno de estos vicerrectorados está presidido por una mujer: Vicerrectorado de Estudiantes y Asuntos Sociales. Podemos hablar claramente de una doble segregación: vertical y horizontal. Vertical porque son muy pocas las docentes que alcanzan puestos de gestión y horizontal porque nos parece que no es casualidad que sea éste un vicerrectorado femenino. Nuevamente encontramos cómo se asigna a las mujeres aquellas actividades consideradas como una extensión del trabajo familiar. 
Cada vicerrectorado cuenta con una serie de Secretariados. Su número varía en función de las necesidades. Dado que son cargos dependientes del/a vicerrector/a, podríamos suponer que encontraríamos un número elevado de mujeres, ya que son puestos en los que predominan las relaciones de subordinación (segregación vertical). Pues bien, si hay un total de 33 Secretariados, tan sólo el 27,26\% están ocupados por mujeres. Comprobamos, una vez más, que el mayor número de direcciones de Secretariados ocupados por mujeres son los pertenecientes al Vicerrectorado de Estudiantes y Asuntos Sociales. Este dato corrobora la existencia de la segregación horizontal: las mujeres se aglutinan en las tareas o actividades que tienen una relación directa con el cuidado de los demás, en este caso de los más jóvenes. Destacar también que éste es uno de los vicerrectorados que mayor número de secretariados tiene, lo que favorece la elección de mujeres para ocuparlos. Además, si este vicerrectorado está regido por una mujer puede que ésta tenga confianza en otras mujeres. Quizás podamos empezar a hablar del establecimiento de redes de confianza entre las mujeres y de una cierta complicidad que permita que cada vez un mayor número de mujeres alcance puestos de responsabilidad en la ULE.

Pero todavía encontramos que algunos varones que ocupan cargos de responsabilidad prefieren delegar parte de sus funciones en otros varones, como se ha venido haciendo tradicionalmente. $\mathrm{Y}$ así lo constatamos en cuatro de los diez Vicerrectorados, donde no aparece ningún nombre femenino.

c) Secretario General. (Capítulo II, Sección 2a , Subsección 3a , Estatuto ULE). Es la persona encargada de auxiliar al rector en las tareas de organización y régimen académico. Será nombrado por el rector entre funcionarios pertenecientes a cuerpos del grupo A que presten sus servicios en la Universidad (Artículo 82). En la ULE, este cargo está ocupado por una mujer, la cual propuso para el cargo de Vicesecretarios General a un varón, que la auxiliará en sus funciones y la podrá sustituir en caso necesario. El Secretario General propone al rector el nombramiento del Vicesecretario entre funcionarios del grupo A de la Universidad. 
Educación superior y segregación de género: órganos unipersonales...

d) Gerente. (Capítulo II, Sección $2^{\mathrm{a}}$, Subsección $4^{\mathrm{a}}$, Estatuto ULE). Será propuesto por el rector y nombrado por éste de acuerdo con el Consejo Social, entre titulados superiores. Debe gestionar los servicios administrativos y económicos de la Universidad, bajo la supervisión del rector. El gerente puede ser auxiliado por uno o más vicegerentes (Artículo 83). La gerencia de la ULE está presidida por un varón que, a su vez, se siente apoyado por dos vicegerentes: un hombre y una mujer. Sólo pueden ocupar estos puestos las personas con titulación superior.

\section{Mujeres en los órganos unipersonales de las Facultades y Escuelas de la ULE}

La Universidad cuenta con ocho Facultades y cuatro Escuelas universitarias, en las que se imparten titulaciones de primer y segundo ciclo. El primer grupo está formado por:

- Facultad de Veterinaria.

- Facultad de Ciencias Biológicas y Ambientales.

- Facultad de Derecho.

- Facultad de Filosofía y Letras.

- Facultad de Ciencias del Trabajo.

- Facultad de Educación.

- Facultad de Ciencias de la Actividad Física y del Deporte.

El segundo grupo lo componen:

- Escuela de Ingenierías Industrial e Informática.

- Escuela Superior y Técnica de Ingenieros de Minas.

- Escuela Superior y Técnica de Ingeniería Agraria.

- Escuela Universitaria de Ciencias de la Salud.

Además tiene adscritos tres centros, que no tendremos en cuenta para nuestro análisis. 
Las Facultades y Escuelas son los centros encargados de la organización de las enseñanzas y de los procesos académicos, administrativos y de gestión conducentes a la obtención de títulos de carácter oficial y validez en todo el territorio nacional, títulos propios, así como de otras funciones que determine el Estatuto (Título Primero, Capítulo II, Artículo 8, Estatuto ULE).

Cada uno de estos centros está gobernado por un Decano o un Director, que es la persona encargada de representar al centro y ejercer las funciones de dirección y gestión del mismo. El Decano de Facultad o Director de Escuela es elegido por la Junta de Centro, entre aquellos de sus miembros que sean profesores doctores pertenecientes a los cuerpos docentes universitarios. En su defecto, en las Escuelas Universitarias y en las Escuelas Universitarias Politécnicas, el Director será elegido entre funcionarios de cuerpos docentes universitarios no doctores o profesores contratados doctores (Capítulo IV, Sección 2a, Subsección 1a, Artículos 91 y 92, Estatuto ULE).

El Decano y el Director de Escuela pueden proponer al Rector el nombramiento de uno o varios Vicedecanos y de uno o más Subdirectores y de un Secretario para el centro docente. El número de vicedecanos y subdirectores dependerá de las necesidades que la Junta de Centro estime.

Tabla 3

Equipos de dirección de centros: Facultades

\begin{tabular}{|l|l|l|l|l|l|}
\hline Facultades & $\mathrm{V}$ & $\mathrm{M}$ & Total & $\% \mathrm{~V}$ & $\% \mathrm{M}$ \\
\hline Decanos/as & 7 & 1 & 8 & 87,5 & 12,5 \\
${\text { Vicedec. } 1^{\circ}}^{\circ}$ & 6 & 2 & 8 & 75 & 25 \\
Vicedec. 2 $^{\text {o }}$ & 3 & 4 & 7 & 42,86 & 57,14 \\
Vicedec. 3 $^{\circ}$ & 1 & 3 & 1 & 100 & 37,5 \\
Secretarios/as & 5 & & 8 & 62,5 & \\
\hline TOTAL & 22 & 10 & 32 & 68,75 & 31,25 \\
\hline
\end{tabular}

Fuente: Universidad de León. Elaboración propia 
Educación superior y segregación de género: órganos unipersonales...

Tabla 4

Equipos de dirección de centros: Escuelas Universitarias.

\begin{tabular}{|l|l|l|l|l|l|}
\hline Escuelas & $\mathrm{V}$ & $\mathrm{M}$ & Total & $\% \mathrm{~V}$ & $\% \mathrm{M}$ \\
Universitarias & & & & & \\
\hline Director/a & 4 & 2 & 4 & 100 & \\
Subdire. $^{\mathrm{o}}$ & 2 & & 4 & 50 & 50 \\
Subdire. $^{\mathrm{o}}$ & 4 & & 4 & 100 & \\
Subdire. $^{\mathrm{o}}$ & 3 & & 3 & 100 & \\
Secretario/a & 4 & & 4 & 100 & \\
\hline TOTAL & 17 & 2 & 19 & 89,46 & 10,54 \\
\hline
\end{tabular}

Fuente: Universidad de León. Elaboración propia

Todos los Centros de la ULE están dirigidos por varones, excepto la Facultad de Ciencias Económicas y Empresariales. Esto quiere decir que sólo un 8\% de las Facultades y Escuelas tienen dirección femenina. Ni siquiera los centros de enseñanza tradicionalmente feminizados, como es el caso de la Facultad de Filosofía y Letras, la Facultad de Educación o la Escuela Universitaria de Ciencias de la Salud, están encabezados por una mujer.

Si analizamos la composición del personal auxiliar, destacamos que el $29 \%$ está formado por mujeres que realizan tareas de vicedecanas, subdirectoras o secretarias. Comprobamos que tanto en las tareas de dirección como en las delegadas de dirección, la representación femenina no llega al umbral mínimo establecido por la Unión Europea para alcanzar la paridad en la participación política y social de las mujeres.

Los Vicedecanos o Subdirectores tienen como misión fundamental dirigir y coordinar, por delegación y bajo la autoridad del Decano o Director, las actividades que éste les asigne. Serán propuestos entre los profesores con dedicación a tiempo completo pertenecientes a la Junta de Facultad o Escuela (Capítulo IV, Sección 2a, Subsección 2a Artículo 94, Estatuto ULE). 
El Secretario, entre otras atribuciones, debe auxiliar al Decano o Director en sus funciones. Será propuesto de entre los profesores con dedicación a tiempo completo, los ayudantes y el personal de administración y servicios vinculados o adscritos al Centro (Capítulo IV, Sección 2a, Subsección $3^{\mathrm{a}}$, Artículo 95, Estatuto ULE).

En términos globales, las mujeres representan algo más del $30 \%$ en los cargos de dirección de las Facultades. Sin embargo, su presencia en las Escuelas Universitarias es del 10,54\%. Esto se debe principalmente al peso que tienen en este grupo de centros docentes las Escuelas de ingeniería Técnica y Superior.

Queremos destacar el caso de la Facultad de Filosofía y Letras porque no cuenta con ninguna mujer en su equipo de dirección y gestión. Nos sorprende menos no encontrar mujeres en la Escuela Superior y Técnica de Ingenieros de Minas y en la Escuela Superior y Técnica de Ingeniería Agraria. Las Ingenierías, tradicionalmente han sido espacios masculinos, tanto entre el alumnado como entre el profesorado. Aunque, en los últimos años parece que cuando relacionamos el número de alumnas con el número de profesoras en estos centros, es más ventajoso para estas últimas que en otros centros tipificados como femeninos. Sin embargo, el poder, es decir, la dirección y gestión sigue estando en manos masculinas (García de Cortázar, M.L.; García de León, M.A., 1997).

5. Mujeres en los órganos unipersonales de los Departamentos. Distinguimos las siguientes figuras:

- Director/a (Capítulo VI, Sección 2a, Subsección 1 ${ }^{\mathrm{a}}$, Artículos 102, 103, 104, Estatuto ULE). El Director del Departamento ostenta la representación del Departamento ejerciendo las funciones de dirección y gestión del mismo. Es nombrado por el Rector y elegido por el Consejo de Departamento entre los profesores funcionarios doctores pertenecientes al mismo. El Director estará acompañado en sus tareas por el Subdirector y el Secretario que serán propuestos por él mismo.

- Subdirector/a (Capítulo VI, Sección 2a, Subsección 2a, Artículo 105, Estatuto ULE). El Subdirector será propuesto al Rector por el Director de Departamento, entre los 
Educación superior y segregación de género: órganos unipersonales...

profesores con dedicación a tiempo completo pertenecientes al Consejo de Departamento.

- Secretario/a (Capítulo VI, Sección 2a, Subsección 3a, Artículo 106, Estatuto ULE). Será nombrado por el Rector, a propuesta del Director, entre los profesores con dedicación a tiempo completo, los ayudantes y el personal de administración y servicios que formen parte del Departamento.

Tabla 5

Equipo de direcciones de Departamentos

\begin{tabular}{|l|l|l|l|l|l|}
\hline $\begin{array}{l}\text { Equipo de } \\
\text { Direcciones de } \\
\text { Departamentos }\end{array}$ & $\mathrm{V}$ & $\mathrm{M}$ & Total & $\% \mathrm{~V}$ & $\% \mathrm{M}$ \\
\hline Directores/as & 26 & 7 & 33 & 78,77 & 21,23 \\
Subdirectores/as & 17 & 14 & 31 & 54,82 & 45,18 \\
Secretarios/as & 13 & 19 & 32 & 40,62 & 59,38 \\
\hline TOTAL & 56 & 40 & 96 & 58,32 & 41,68 \\
\hline
\end{tabular}

Fuente: Universidad de León. Elaboración propia

La Universidad de León cuenta con 33 Departamentos, pero sólo 7 están dirigidos por una mujer. Los Departamentos dirigidos por mujeres son: Ciencias Jurídicas Básicas; Derecho de la Administración y Relaciones Internacionales; Derecho Privado; Ecología, genética y microbiología; Enfermería y Fisioterapia; Filosofía y Ciencias de la Educación y Patrimonio histórico artístico y de la cultura escrita.

Si hacemos un análisis global de los datos, destacamos la existencia de paridad en los equipos de dirección de departamentos (58,32\% varones y $41,68 \%$ mujeres). Sin embargo, un análisis detallado nos indica, por un lado, la ausencia de profesoras en la Dirección de Departamento $(21,23 \%)$. Este dato muestra, una vez más, la segregación vertical existente en la Universidad. Por otro lado, comprobamos cómo la presencia de mujeres aumenta a medida que el estatus es más dependiente o más subordinado. Se produce una segregación horizontal: las mujeres se concentran, sobre todo, en el nivel 
de Secretario/a de Departamento. Parece indicar que tanto los Directores como las Directoras de Departamento confían más las tareas de Secretaría a las mujeres. Este hecho muestra el arraigo social que tiene el estereotipo de mujer como organizadora del trabajo.

\section{Conclusiones}

Las mujeres representan el $38,15 \%$ del profesorado de la Universidad de León..Esto significa que hay más profesores tanto entre el cuerpo de funcionarios universitario como entre el profesorado contratado.

Por categoría docente, apreciamos una segregación vertical: a mayor categoría, menor número de mujeres. Así, la proporción de catedráticas no llega al 16\% del profesorado que ocupa este estatus. Esto tiene gran relevancia, no sólo para el futuro de la Universidad, sino también para el futuro de las propias docentes. La legislación universitaria prevee que para ocupar algunos cargos de gestión, por ejemplo, el de rector, sea condición imprescindible ocupar este escalafón.

Observamos la existencia del "techo de cristal", es decir, en la Universidad hay una barrera invisible que impide que las docentes alcancen el rango más alto dentro de la carrera académica. Así lo prueba el hecho de que más del 45\% del Profesorado Titular de Universidad y más del 35\% de la categoría de Catedráticos/as de Escuela Universitaria son mujeres. Parece que hay algo que impide que las mujeres den ese salto hacia arriba de la pirámide.

Encontramos segregación vertical en el reparto de cargos de responsabilidad y gestión, reflejándose un desequilibrio entre varones y mujeres. Existe subrepresentación femenina en los órganos unipersonales de gobierno de la Universidad. El Pabellón de Gobierno de la universidad está ocupado principalmente por varones: rector, vicerrectores y direcciones de secretariado. Tan sólo una mujer está al frente de un Vicerrectorado: Estudiantes y Asuntos Sociales. Ella hace posible que otras mujeres 
Educación superior y segregación de género: órganos unipersonales...

ocupen direcciones de secretariado: es el vicerrectorado que cuenta con mayor número de mujeres.

Pero las mujeres también están ausentes en los órganos unipersonales de las Facultades y Escuelas Universitarias (31,25\% y 10,54\%, respectivamente).

Lo mismo podemos decir de la representación femenina en los órganos unipersonales de los Departamentos, aunque estos datos son más optimistas. La participación femenina es del $41,68 \%$. Nos parece importante esta cifra porque la participación femenina en estos puestos de responsabilidad puede ser un primer paso para que en el futuro las docentes sigan escalando puestos en la jerarquía universitaria. 


\section{BIBLIOGRAFÍA}

Almarcha Barbado, A.; GonzÁlez Rodríguez, B.; GonzÁlez Jorge, C. (1994): Cambio y desigualdad en el profesorado universitario. Reis, 66.

Antón Sevilla, S. (2005): Académicas en la Universidad de Alicante. Alicante: Centro de Estudios sobre la Mujer.

BOE, $\mathrm{n}^{\circ}$ 307. Lunes 24 de diciembre 2001.

BOULE, $n^{\circ} 8$. Octubre de 2003.

CIDE/Instituto de la Mujer (2001): Las mujeres en el sistema educativo. Madrid: Instituto de la Mujer.

Consejo Económico y Social (2003): Segundo Informe sobre la situación de las mujeres en la realidad sociolaboral española. Madrid: Colección Informes CES. $\mathrm{N}^{\mathrm{o}}$ 3/2003.

Cordero del Campillo, M. (1990): Universidad de León. El primer decenio 19791989. León: Secretariado de Publicaciones, Universidad de León.

FERnÁNDEZ VillanueVA, C. (1989): Las mujeres en la Universidad española: docencia, investigación y poder. Datos y aspectos cualitativos. Revista de Educación, no 290.

GARcíA de CORTÁZAR, M.L.; GARCÍA DE LEÓN, M.A. (1997): Mujeres en minoría. Una investigación sociológica sobre las catedráticas de universidad en España. Madrid: CIS. Opiniones y Actitudes, $\mathrm{n}^{\circ} 16$.

García de EnTERríA, E. y Escalante, J. A. (1987): Legislación Administrativa Básica. Madrid: Editorial Civitas, S.A.

García de LeÓn, M. A.; GARcía de CORTÁzAR, M.L. (1992): Universidades y universitarios (1970-1990). Revista de Educación. Extraordinario.

Instituto de la Mujer (2001): Las mujeres en cifras. 1996-2000. Madrid: Instituto de la Mujer.

Instituto de la Mujer (2001): Las académicas. (Profesorado universitario y género). Madrid: Instituto de la Mujer. 
Educación superior y segregación de género: órganos unipersonales...

Ministerio de Trabajo y Asuntos Sociales (2003): IV Plan de Igualdad de Oportunidades entre Mujeres y Hombres. Madrid: Ministerio de Trabajo y Asuntos Sociales.

RocA I TRIA, E. (1995): Mujer y poder en la Universidad. Oviedo: Seminario de Estudios de la Mujer. Vicerrectorado de Investigación. Universidad de Oviedo. 
